\title{
Selective non-operative management of Penetrating Thoracic Injury
}

Oscar J.F. Van Waes ${ }^{1}$, Jens A. Halm ${ }^{1}$, Diederik I. Van Imhoff $^{1}$, Pradeep H. Navsaria ${ }^{2}$, Andrew J. Nicol ${ }^{2}$, Michael H.J. Verhofstad ${ }^{1}$, Jefrey Vermeulen ${ }^{1,3}$

${ }^{1}$ Trauma Research Unit Department of Surgery, Erasmus MC, University Medical Center Rotterdam, Rotterdam, The Netherlands

${ }^{2}$ Trauma Center, Groote Schuur Hospital, University of Cape Town, Cape Town, South Africa

${ }^{3}$ Department of Surgery-Traumatology, Spaarne Gasthuis, Haarlem, The Netherlands

Corresponding author:

Oscar J.F. Van Waes, MD

Erasmus MC, University Medical Center Rotterdam

Trauma Research Unit Department of Surgery, Room H-822k

P.O. Box 2040, 3000 CA Rotterdam, The Netherlands

Tel: + 31107031050

Fax: +31 107032396

E-mail: o.vanwaes@erasmusmc.nl

Conflicts of interest: None declared

Funding source: No funding was obtained for this study

Category: original article; no data has been presented or published previously 


\section{ABSTRACT}

Background Thoracic penetrating injury is cause for up to a fifth of all non-natural deaths. The aim of this study was to determine the success of selective non-operative management (SNOM) of patients presenting with a penetrating thoracic injury (PTI).

Methods A prospective study of patients with PTI who presented to a level 1 Trauma Center between April 2012 and August 2012.

Results 248 patients were included in the study with $5.7 \%(n=14)$ requiring immediate emergency surgery. Overall 5 of these 248 patients died resulting in a mortality rate of $2.0 \%$. Primarily 221 patients (89.1\%) were managed with SNOM of whom 15 (6.8\%) failed conservative management. Failure of SNOM was primarily caused by complications of chest tube drainage ( $n=12)$ (e.g. retained clot, empyema) and delayed development of cardiac tamponade $(\mathrm{n}=3)$. The survival rate in the SNOM group was $100 \%$.

Conclusion PTI has a low in-hospital mortality rate. Only $16.5 \% \quad(41 / 248)$ of the patients presenting with PTI will need surgical treatment. The other patients are safe to be treated conservatively according to a protocolised SNOM approach for PTI without any additional mortality. Conservative treatment of patients that were selected for this non-operative treatment strategy with repeated clinical re-assessment was successful in $93.2 \%$. 


\section{INTRODUCTION}

Thoracic penetrating injury causes up to a fifth of all non-natural deaths (1-3). The number of patients suffering from penetrating thoracic injuries (PTI) is low in Western Europe compared to the urban trauma centers of the USA or South Africa (4-8). Studies of treatment strategies in penetrating trauma of the chest are rare. Based on the experience with penetrating trauma in a high volume tertiary referral center for penetrating trauma, we have initiated a penetrating thoracic injury treatment protocol on which we have previously reported (9). In the current study the success rate of selective non-operative management (SNOM) using protocolled management strategies and the survival after PTI were prospectively analyzed. 


\section{METHODS}

All consecutive patients with PTI presenting to the Trauma Center of the Groote Schuur Hospital, Cape Town, from April 1 through August30, 2012 were included in this prospective observational study. Approval for data collection was obtained from local research ethics committee. Age, gender, mechanism and type of injury, New Injury Severity Score (NISS), clinical manifestations including pre-hospital and presenting vital signs, additional investigations, treatment strategy and outcome of all patients were collected and analyzed. All patients were managed and treated according to an institutionalized protocol for PTI (Figure 1). All calculations and statistical analyses were performed using SPSS statistics version 21.0. Continuous data were non-parametric, and are presented as median with $\mathrm{P}_{25}-\mathrm{P}_{75}$. Categorical data are shown as numbers with percentages. Statistical significance of difference between $\mathrm{p}$ $<0.001$ and $\mathrm{p}<0.05$ was assessed using a Mann-Whitney U-test for continuous data and a Chi-Squared test or Fisher Exact test for categorical data.

\section{Protocol for management of penetrating thoracic injury}

All patients were considered at risk for a PTI if they sustained stab wounds (SW) ranging cranially from neck, the sternum or clavicles to caudally the level of the twelfth rib on the anterior side of the patient and posteriorly from the lower neck to twelfth rib. For gunshot wounds (GSW) these margins were increased judiciously (including the groin region anteriorly and buttocks posteriorly) to account for bullet-track-injury. Special attention was paid to inspection of body creases and folds. If the patients were suspected for PTI according to the abovementioned definitions they were assessed and resuscitated according to the ATLS $^{\circledR}$ guidelines (10) and subsequently managed according to the local PTI protocol.

A resuscitative anterolateral emergency department thoracotomy (EDT) was indicated for patients who presented with loss of signs of life (SOL) but with a witnessed cardiac arrest less 
than 15 minutes before. Witnessed loss of SOL in the ED or a persistent systolic hypotension $<60 \mathrm{mmHg}$ (severe shock) despite aggressive resuscitation also warranted an EDT. All hemodynamically stable patients (systolic $>90 \mathrm{mmHg}$ ) and patients stabilized after resuscitation from moderate shock (systolic BP >60 mmHg and $<90 \mathrm{mmHg}$ ) underwent a plain chest X-ray as part of ATLS ${ }^{\circledR}$ protocol. Chest drainage of more than $1500 \mathrm{ml}$ of blood immediately or more than $200 \mathrm{ml}$ per hour over the next several hours accompanied by hemodynamically instability was an indication for urgent thoracic surgery (UTS).

Computed tomography angiography (CTA) was indicated for patients with a SW with significant drainage $(>500 \mathrm{ml})$ directly after insertion of chest drain to assess whether or not the injury was suitable for SNOM. All hemodynamically stable patients with a GSW underwent additional CTA to exclude a mediastinal injury.

Signs of a pericardial effusion on chest X-ray (e.g. globular heart, straightened left heart border) needed confirmation by CTA or trans-thoracic ultrasound (US). Patients with clinical signs of tamponade underwent urgent median sternotomy. Patients with pericardial effusion without clinical signs of tamponade were planned for drainage through a subxyphoid window (SXW) after 24-hours. These patients were admitted to the high-care trauma ward for hemodynamic and airway monitoring as well as clinical wound and chest drainage examination every four hours.

There were four different types of surgical intervention. 1. A resuscitative anterolateral left sided thoracotomy performed in the shock room at the emergency department (EDT). 2. Urgent thoracic surgery (UTS) was performed for patients responding to resuscitation with vital parameters (e.g. systolic blood pressure above $60 \mathrm{mmHg}$ ), which allowed transport to the operation theater for definite care of life-threatening injuries, e.g. an urgent SXW or massive blood loss after chest drainage. 3. A planned SXW was performed after 24-hours in hemodynamically stable patients with pericardial effusion. 4. The last group consisted of 
patients who required late ( $>48$ hours) surgical treatment such as video assisted thoracic surgery (VATS) or thoracotomy for retained clot, empyema or persistent air leakage as well as SXW procedure for delayed pericardial tamponade. 


\section{RESULTS}

\section{Demographics}

Over a 5-month period 425 patients were presented with chest injuries of which 259 patients were diagnosed with PTI. Of these 259 patients 188 presented with stab wounds, 66 with one or more gunshot wounds, and five patients with a combination of both GSW and SW. Eleven patients deceased in the ED from associated injuries: 6 of these suffered from un-survivable traumatic brain injury and five died from a major extra thoracic vascular injury not responding to resuscitation. These patients were excluded from the study, leaving 248 patients included for analysis (Figure 2).

The patients with PTI had a median age of 27 years $\left(\mathrm{P}_{25}-\mathrm{P}_{75}\right.$ 21-32) and 239 (96.4\%) patients were males. Nearly half of the patients $(n=124)$ presented with isolated injury to the chest. Associated injuries were predominantly to the head, neck and face $23.4 \%(n=58)$, extremities 19.8\% $(n=49)$ and abdomen $17.7 \%(n=44)$.

Most patients ( $\mathrm{n}=157)$ were referred from local community medical facilities or non-trauma specialized hospitals (63.3\%). The remainder of patients was transferred directly from the accident scene. Seven out of the eleven patients excluded from this study that died at the ED due to others injuries then PTI were referred from another medical facility. Three from the five patients, who died during treatment of their PTI, were referrals. The point of origin proved not significantly different (directly from injury scene versus referral) $(p=1.000)$. Of the patients requiring admission, $70.6 \% \quad(n=175)$ underwent chest tube drainage for a hemopneumothorax. In 51 patients (20.6\%), fitted with a chest tube prior to arrival on the ED, an additional thoracic drain was placed on the ED. Most patients arrived at the ED with normal blood pressures. Only 5 patients (2.2\%) were in severe shock, and 26 (11.6\%) in moderate shock upon arrival at the ED. From 23 patients no initial systolic blood pressure was recorded. None of the patients with severe shock died, $7.7 \%$ of patients with moderate shock 
(2 out of 26) died, whereas only $1 \%(n=2)$ of normotensive patients eventually died. These differences were not found to be statistically significant $(\mathrm{p}=0.052)$.

\section{Selective non-operative management (SNOM)}

Of all patients with PTI 89.1\% (n=221) were primarily treated with SNOM.

SNOM treatment failed in $6.8 \%$ of these patients $(n=15)$. Eight patients required video assisted thoracic surgery (VATS) to treat late onset PTI complications: empyema $(n=3)$, clotted hemothorax $(n=4)$, and a combination of persistent air leakage with empyema $(n=1)$. CT scan confirmed the need for VATS in these eight patients. Two patients developed thoracic sepsis and were treated by wash out of the chest through thoracotomy. Two other patients underwent a thoracotomy for massive residual cloth removal. Three patients, who had already been discharged from hospital for several days, returned to the ED with signs of cardiac tamponade and needed to undergo SXW procedure for drainage. One of them revealed a positive SXW and hence needed conversion to a sternotomy with myocardial repair. None of the patients primarily selected for SNOM died and therefore overall survival of the SNOM group was 100 percent.

The median NISS was significantly lower, $9\left(\mathrm{P}_{25}-\mathrm{P}_{75}\right.$ 5-13) for the patients in whom SNOM was successful $(n=206)$ compared with those who required surgical intervention $25\left(\mathrm{P}_{25}-\mathrm{P}_{75}\right.$ 18-32) $(\mathrm{n}=41) \mathrm{p}<0.0001$.

\section{Surgical interventions}

Results of surgical interventions are listed in Table 1. Six patients underwent EDT, because of loss of signs of life $(n=4)$ or because of being non-responders to resuscitation $(n=2)$. Three patients that underwent EDT survived (50\%). Two survivors were successfully treated for cardiac tamponade, which caused loss of SOL. One patient, in extremis, survived thoracic 
hemorrhage by clamping the lung hilum after which definitive surgical treatment of the lung injury was performed in the operating room. A fourth non-responder to initial resuscitation did not receive an EDT, since the treating physician deemed a massive hemothorax nonsurvivable.

UTS was performed in eight patients and comprised six sternotomies, $1 \mathrm{SXW}$, and 1 thoracotomy (Table 1). One patient died after emergency left-sided thoracotomy for unexpected asystole while performing neck exploration for penetrating injury. The thoracotomy did not reveal any lethal intra-thoracic injury. Six sternotomies were performed. Three patients required sternotomy for proximal vascular control. In two patients exhibiting signs of cardiac tamponade, sternotomy revealed a penetrating cardiac injury, which could be successfully sutured. One patient with suspected cardiac tamponade underwent a negative sternotomy. A SXW procedure was performed as UTS in a patient after a transthoracic GSW resulting in a pneumohemo-pericardium. After evacuating $50 \mathrm{ml}$ of blood, no ongoing bleeding was noted and was consequently treated by drainage alone and observation.

Twelve patients with diagnosed pericardial effusion, but without signs of tamponade, were planned for SXW procedure after 24- hours according the local protocol. Six patients awaiting their planned SXW displayed acute clinical deterioration on the ward (hypotension or tachycardia) and therefore underwent acute SXW. One of these patients needed conversion to sternotomy, which revealed injury to the right ventricle amiable for suture-repair. The other six patients could be treated by SXW pericardial drainage alone and close observation in the high care ward afterwards. After an uneventful observation of twelve hours the pericardial drain was removed in all patients without complications. All 12 patients who were initially planned for delayed SXW survived. 


\section{DISCUSSION}

This study displayed that patients suffering from PTI who reach the ED with signs of life have an excellent change of survival (98\%), with only 1 out of 10 patients requiring surgical intervention. Only 15 patients (6.8\%) failed their initial SNOM but without additional mortality.

Pre-hospital mortality for patients with PTI is as high as 75\% (11). In contrast, in-hospital outcome after PTI is reported to be very good (12). The in-hospital mortality after PTI was only $2.0 \%(5 / 248)$ in this study. A selection bias might be suggested since a large number of patients (63.3\%) are referred from outside care facilities. In Europe and America most patients are directly transferred from the incident scene to the ED of the nearest trauma center without a primary assessment and treatment in a local medical $(3,7,9)$. Point of origin was however not significantly different in this study for the deceased patients. A possible other explanation for the high in-hospital survival rate in this study may be the relative high incidence of SW (75\%), as compared to other studies in which up to $60 \%$ of all PTI patients are injured by GSW (2,12-14).

This study confirms that most PTI could be treated conservatively by SNOM principles as has been published (3,5,11-13). Eighty-three percent $(n=206)$ could be managed successfully by observation alone, possibly with chest drainage as only treatment.

Using ATLS ${ }^{\circledR}$ guidelines and an institutionalized protocol, PTI patients were selected for SNOM or necessary surgical treatment. CTA was the diagnostic adjunctive of choice in hemodynamically stable patients with PTI due to GSW's or with a significant hemopneumothorax. CTA has an excellent sensitivity of $95 \%$ and predictive value of $98 \%$ to rule out occult injuries that might impede SNOM (15-17). SNOM had a success rate of 93.2\%. Only fifteen patients failed SNOM, because of the late onset of complications, which could be treated subsequently without any mortality. 
The use of VATS for the treatment of persistent clot and for washout of pleural empyema has been widely advocated (18-21). Eight patients initially treated non-operatively needed to undergo VATS to treat late onset complications after PTI. VATS procedure led to an uneventful recovery. Two of the patients in which SNOM failed had already been discharged from hospital but returned to the ED several days later because of new signs of cardiac tamponade and needed treatment.

Delayed cardiac tamponade is a potentially lethal complication of PTI. Being a rare entity it has only been described in small series or case reports (22-24). The question is whether all patients with PTI need to undergo standard additional diagnostic investigations such as transesophageal ultrasound or CTA to rule out pericardial effusion. Three patients with a pericardial effusion were missed. One admitted and two already discharged without noted pericardial effusion. Trans-esophageal ultrasound is less suitable for repetitive screening for pericardial effusion since the patient usually needs to be sedated (25-27). Although US is advocated as an accurate and sensitive tool to detect a hemopericardium (28-31) the presence of hemothorax and/or pneumopericardium confounds the interpretation of US and may lead to high number of false negative investigations. In case of equivocal US results or the presence of a hemothorax, CT is the adjunctive of choice to screen for occult penetrating cardiac injuries or repeat US at 24 hours once the hemothorax has cleared (32). In only $10.4 \%$ of all patients presenting with diagnosed PTI, there was a primary indication for surgical treatment; EDT (2.4\%), UTS (3.2\%), planned SXW (4.8\%). A planned SXW was reserved for those patients with ultrasound (or CT) proven pericardial effusion, but without hemodynamic instability. Nevertheless, in six of the twelve patients with noted pericardial effusion that were initially hemodynamically stable during their stay and management on the ED showed unexpected clinical deterioration on the ward and subsequently needed an acute SXW. None of these six patients suffered additional morbidity after acute SXW. When intensive 
observational care is guaranteed, a planned SXW is a safe management strategy in hemodynamically stable PTI patients with pericardial effusion $(33,34)$. It should be mentioned that in contrast to trauma populations in Western Europe or America, pericardial effusion in South Africa might be caused by tuberculosis, which explains the high rate $(91.7 \%)$ of negative planned SXW (35).

Although potentially lethal as shown in prior research, PTI has a low in hospital mortality rate of $2.0 \%$ (three EDT, one UTS, and one abstained patient) in this series. A judicious use of clinical protocol and adjuncts will render a high success rate of SNOM (93.2\%) thereby limiting unnecessary surgical explorations with possible (iatrogenic) complications. On the other hand aggressive surgical resuscitation including low threshold for EDT might contribute to this high survival rate for PTI.

\section{CONCLUSION}

PTI has a low in-hospital mortality rate of only $2.0 \%$. Only $16.5 \%(41 / 248)$ of the patients presenting with PTI will need surgical treatment. The other patients are safe to be treated conservatively according to a protocolised SNOM approach for PTI without any additional mortality. Conservative treatment of patients that were selected for this non-operative treatment strategy with repeated clinical re-assessment was successful in $93.2 \%$. 


\section{FIGURES}

Figure 1. Summary of local protocol for resuscitation and initial treatment of patients sustaining penetrating thoracic injury

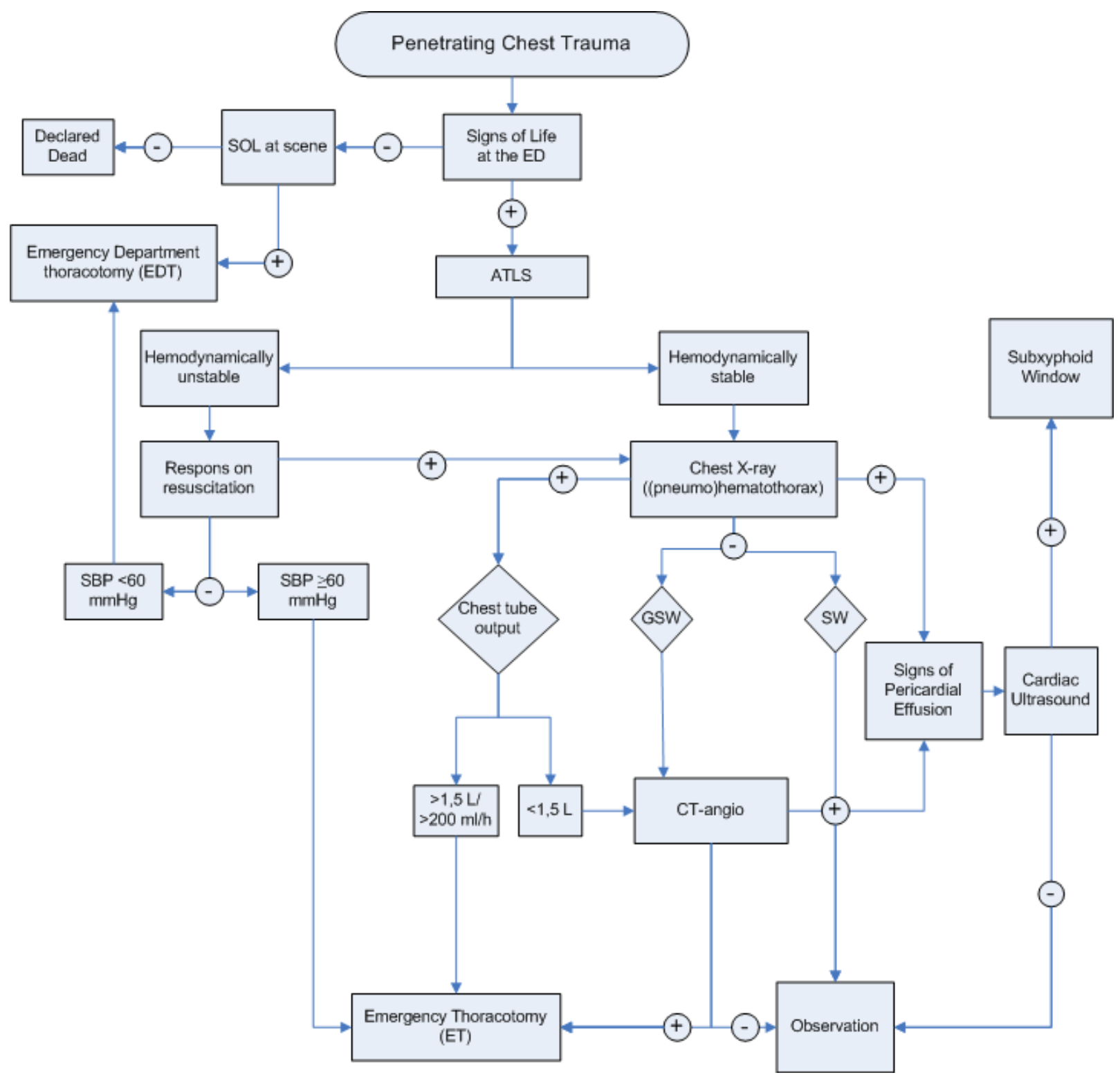

SOL=signs of life; ED=emergency department; $A T L S^{\circledR}=$ advanced trauma life support; $\mathrm{SBP}=$ systolic blood pressure; GSW=gunshot wound; SW=stab wound; CT=computed tomography 
Figure 2. Study inclusion flowchart

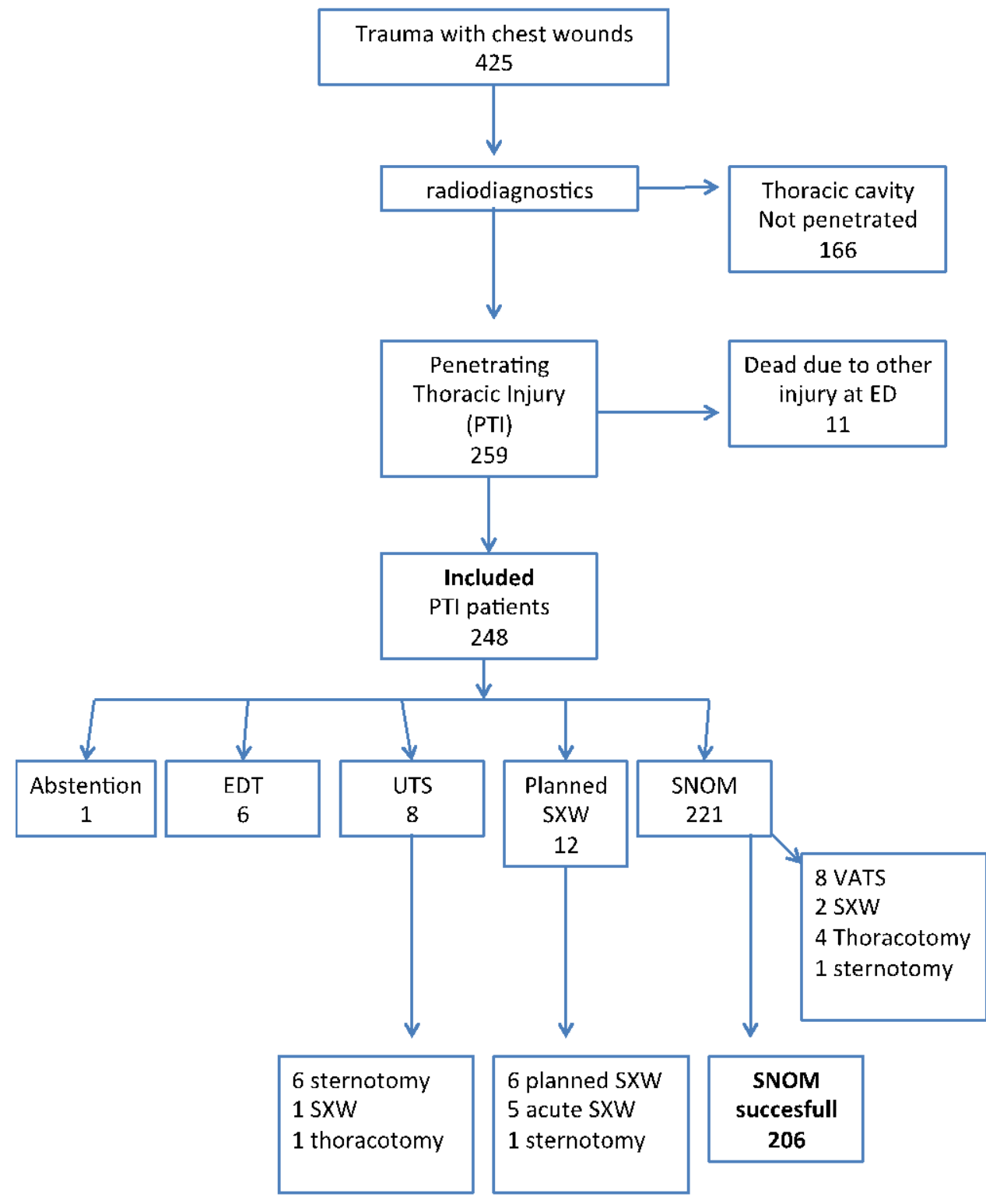

ED=emergency department; SXW=subxyphoid window; VATS=video assisted thoracoscopy

\section{TABLES}

Table 1. Emergency and planned surgical procedures for patients after PTI 


\begin{tabular}{|c|c|c|c|c|}
\hline Procedure & Sub type & Indication & Findings / treatment & Survival \\
\hline $\begin{array}{l}\text { Emergency department } \\
\text { thoracotomy (EDT) } \\
(\mathrm{n}=6)\end{array}$ & $\begin{array}{l}\text { Anterolateral } \\
\text { thoracotomy } \\
(\mathrm{n}=6)\end{array}$ & $\begin{array}{l}\text { Loss of SOL }(\mathrm{n}=4) \\
\text { Non-responder to } \\
\text { resuscitation (n=2) }\end{array}$ & $\begin{array}{l}\text { Tamponade with myocardial injury with } 2 \mathrm{x} \\
\text { successful suture repair } 1 \mathrm{x} \text { unsuccessful,, lung } \\
\text { hilum successful clamped (n=1) } \\
\text { Gross cardiovascular injury beyond repair } \\
(\mathrm{n}=2)\end{array}$ & $50 \%$ \\
\hline $\begin{array}{l}\text { Urgent Thoracic } \\
\text { Surgery (UTS) }(n=8)\end{array}$ & $\begin{array}{l}\text { SXW (n=1) } \\
\text { Thoracotomy } \\
\text { (n=1) }\end{array}$ & $\begin{array}{l}\text { Subclavian artery injury } \\
\text { control (n=1) } \\
\text { medinastinal vascular injury } \\
(\mathrm{n}=2) \\
\text { cardiac injury (n=2) } \\
\text { shock with suspected } \\
\text { pericardial effusion ( } \mathrm{n}=1) \\
\text { Exploratory SXW for } \\
\text { transthoracic GSW (n=1) } \\
\text { Severe shock with neck and } \\
\text { thoracic SW, died in theater } \\
\text { (n=1) }\end{array}$ & $\begin{array}{l}\text { Subclavian artery injury repair }(\mathrm{n}=1) \\
\text { Primary vascular repair (n=2) } \\
\text { Primary cardiac suture repair (n=2) } \\
\text { Rinsing pericardium without repair (n=1) } \\
\text { Rinsing pericardium without repair (n=1) } \\
\text { Negative exploratory thoracotomy for } \\
\text { asystole during neck exploration (n=1) }\end{array}$ & $87.5 \%$ \\
\hline Planned SXW $(n=12)$ & & $\begin{array}{l}\text { Pericardial effusion without } \\
\text { clinical signs }(\mathrm{n}=6) \\
\text { Clinical deterioration while } \\
\text { planned for SXW }(\mathrm{n}=5) \\
\text { Sternotomy }(\mathrm{n}=1)\end{array}$ & $\begin{array}{l}\text { Rinsing pericardium without repair }(\mathrm{n}=6) \\
\text { Rinsing pericardium without repair }(\mathrm{n}=5) \\
\text { Hemopericardium conversion of } \mathrm{SXW} \text { to } \\
\text { sternotomy for ventricular repair }(\mathrm{n}=1)\end{array}$ & $100 \%$ \\
\hline $\begin{array}{l}\text { Surgery for } \\
\text { complications after } \\
\text { SNOM } \\
(\mathrm{n}=15)\end{array}$ & $\begin{array}{l}\text { VATS }(\mathrm{n}=8) \\
\text { Thoracotomy } \\
(\mathrm{n}=4) \\
\text { SXW (n=2) } \\
\text { Sternotomy }(\mathrm{n}=1)\end{array}$ & $\begin{array}{l}\text { Retained cloth }(\mathrm{n}=5) \text {, } \\
\text { empyema }(\mathrm{n}=3) \text {, air leakage } \\
\text { and empyema }(\mathrm{n}=1) \\
\text { Empyema with sepsis }(\mathrm{n}=2) \\
\text { Large retained cloth }(\mathrm{n}=2) \\
\text { Delayed tamponade }(\mathrm{n}=2) \\
\text { Clinical worsening + CT } \\
\text { pericardial effusion }\end{array}$ & $\begin{array}{l}\text { Irrigation and drainage }(\mathrm{n}=8) \text {, irrigation, } \\
\text { drainage and wedge resection }(\mathrm{n}=1) \\
\text { Decortication with irrigation and drainage } \\
(\mathrm{n}=4) \\
\text { Rinsing pericardium without repair }(\mathrm{n}=2) \\
\text { Successful suture repair }(\mathrm{n}=1)\end{array}$ & $100 \%$ \\
\hline
\end{tabular}

SOL=signs of life; SXW=subxyphoid window; VATS=video assisted thoracoscopy 


\section{REFERENCES}

1. MacKenzie EJ. Epidemiology of injuries: current trends and future challenges. Epidemiol Rev 2000;22(1): 112-9.

2. LoCicero J, 3rd, Mattox KL. Epidemiology of chest trauma. Surg Clin North Am 1989;69(1): 15-9.

3 von Oppell UO, Bautz P, de Groot M. Penetrating thoracic injuries: what have learnt. Thorac Cardio Surg 2000;48:55-61

4 Mattox KJ, Feliciano DV, Burch J, Beall AC, Jordan GL jr, Debakey ME. Five thousand sevenhunderd sixty cardiovascular injuries in 4459 patients. Ann surg 1989;209(6):698-705

5 Asensio JA, Navarro Soto S, Forno W, Roldan G, Petrone P, Gambaro E, Salim A, Rowe V, Demetriades D. Penetrating cardiac injuries: a complex challenge. Surg Today 2001;31:1041-53

6 Soreide K, Soiland H, Lossius HM, Vetrhus M, Soreide JA, Soreide E. Resuscitative emergency thoracotomy in a Scandinavian trauma hospital, is it justified? Injury 2007;38(1):34-42

7 Kandler K, Konge L, Rafiq S, Larsen CT, Ravn J. Emergency thoracotomies in the largest trauma center in Denmark: 10 years experience. Eur J Trauma Emerg Surg 2012;38:151-6

8 Sektion NCS of the German Trauma Society (DGU) / AUC-academy of Trauma Surgery GmbH. TraumaRegister DGU® Annual Report 2013.

9 van Waes OJF, van Riet PA, Van Lieshout EMM, Den Hartog D. Immediate Thoracotomy for penetrating injuries: ten years' experience at a Dutch level I trauma center. Eur J Trauma Emerg Surg 2012;38:543-51 
10 American College of Surgeons Committee on Trauma (2004) Advanced trauma life support program for doctors, 9th edn. American College of Surgeons, Chicago

11 Clarke DL, Quazi MA, Reddy K, Rutherford Thomson S. Emergency operation for penetrating thoracic trauma in a metropolitan surgical service in South Africa. J Thorac Cardio vasc Surg 2011;142:563-8

12 Mandal AK, Sanusi M. Penetrating chest wounds: 24 years experience . World J Surg 2001;25:1145-9

13 Mollberg NM, Tobachnick D, Lin FJ, Merlotti GJ, Varghese TK, Arensman RM, Massad MG. Age-associated impact on presentation and outcome for penetrating thoracic trauma in the adult and pediatric patient populations. J Trauma Acute Surg. 2014;76(2):273-7

14 Onat S, Ulku R, Avci A, Ates G, Ozcelik C. Urgent thoracotomy for penetrating chest trauma: analysis of 158 patients of a single center. Injury. 2010;41(7): 876-880.

15 O' Conner JR, Scalae TM. Penetrating thoracic great vessel injury: impact of admission hemodynamics and preoperative imaging. J Trauma 2010;68(4):834-7

16 de Vries CS, Africa M, Gebremariam FA, van Rensburg JJ, Otto SF, Potgieter HF. The imaging of stab injuries. Acta Radiol. 2010;51(1): 92-106.

17 Nunez DB Jr, Torres-Leon M, Munera F. Vascular injuries of the neck and thoracic inlet: helical CT-angiographic correlation. Radiographics. 2004;24(4):1087-98

18 Lang-Lazdumski L, Mouroux J, Pons et al. Role of thoracoscopy in chest trauma. Am Thorac Surg. 1997;63:327-33

19 Heniford BT, Carrillo EH, Spain DA, Sosa JL, Fulton RL, Richardson JD. The role of thoracoscopy in the management of retained thoracic collections after trauma. Am Thorac Surg. 1997;4:940-3 
20 Billeter AT, Druen D, Franklin SA, Smith JW, Wrightso W, Richardson JD. Videoassisted thoracoscopy as an important tool for trauma surgeons: a systematic review. Langenbecks Arch Surg. 2013;4:515-23

21 Navsaria PH, Vogel RJ, Nicol AJ. Thorascopic evacuation of retained post traumatic hemothorax. Ann Thorac Surg. 2004;78:282-5

22 Harris DG, Janson JT, van Wyk J, Pretorius J, Rossouw GJ. Delayed pericardial effusion following stab wounds to the chest. Eur J Card Thor Surg. 2003;23:473-6

23 Gonzalez Enriquez S, Gonzalez Fernandez C, Rodriguez Entem F, Garagarza JMSJ, Duran RM. Delayed pericardial tamponade after penetrating chest trauma. Eur J Emerg Med. 2005;12:86-8

24 Huis in 't Veld, Halm JA, van Waes OJF. Een late pericardiale tamponade na penetrerend thoraxletsel 'Shaved and saved by the cold blue steel'. Ned Tijdschr Traum. 2012;5:149-52

25 Porembka DT, Johnson DJ, Hoit BD, Reising J, Davis K Jr, Koutlas T. Penetrating cardiac trauma: a perioperative role for transesophageal echocardiography. Anest Analg. $1993 ; 77: 1275-7$

26 Parasramka SV, Ghotkar S, Kendall J, Fabri BM. Use of transoesophageal echocardiography in management of penetrating cardiac injury. Eur $\mathrm{J}$ Echocardiography. 2008;9:563-4

27 ASA task force on perioperative transesophageal echocardiography. Practice guidelines for perioperative transesophageal echocardiography. Anesthesiology. 2010;112:1-13

28 Plummer D, Bunette D, Asinger R, Ruiz E. Emergency department echocardiography improves outcome in penetrating cardiac injury. Ann Emerg Med. 1992;21:709-12 
29 Aaland M, Bryan FC, Sherman R. two dimensional echocardiogram in hemodynamically stable victims of penetrating precordial trauma. Am Surg. 1994;60:412-15

30 Jimenez E, Martin M, Krukenkamp I, Barret J. Subxyphoid pericardiotomy versus echocardiography: a prospective evaluation of the diagnosis of occult penetrating cardiac injury. Surgery. 1990;108:676-86

31 Meyer D, Jessen M, Grayburn P. Use of echocardiography to detect occult cardiac injury after penetrating thoracic trauma: a prospective study. J Trauma. 1995;39:902-9

32 Nicol AJ, Navsaria PH, Beningfield S, Hommes M, Kahn D. Screening for occult penetrating cardiac injuries. Ann Surg. 2015;261:573-8

33 Navsaria PH, Nicol AJ. Haemopericardium in stable patients after penetrating injury: Is subxyphoid pericardial window and drainage enough? Injury. 2005;36:745-50

34 Nicol AJ, Navsaria PH, Hommes M, Ball CG, Edu S, Kahn D. Sternotomy or drainage for hemopericardium after penetrating trauma. Ann Surg. 2013;00:1-5

35 Reuter H, Burgess LJ, Doubell. Epidemiology of pericardial effusions at a large academic hospital in South Africa. Epidemiol Infect. 2005;133:393-99 